\title{
Le tact pédagogique
}

Clermont Gauthier Université Laval (Canada)

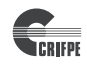

\section{HRONIQUE • Formation des maîtres}

Il y avait à l'époque quelque chose d'utopique, mais aussi de foncièrement généreux chez Comenius qui appelait de ses vœux, au $\mathrm{XVII}{ }^{\mathrm{e}}$ siècle, la création d'un traité de l'art d'enseigner parfaitement tout à tous :

Nous avons l'audace, nous, de promettre une Grande Didactique, je veux dire un traité de l'art complet d'enseigner tout à tous. Et de l'enseigner de telle sorte que le résultat soit infaillible. Et de l'enseigner vite, c'est-à-dire sans aucun dégoût et sans aucune peine pour les élèves et pour les maîtres, mais plutôt avec un extrême plaisir pour les uns et pour les autres. Et de l'enseigner solidement, et non superficiellement et en paroles, mais en promouvant les élèves à la vraie culture scientifique, littéraire et artistique, aux bonnes mœurs, à la piété. (p. 33)

Mais il y a aussi la réalité implacable du terrain qui nous amène à nous représenter les salles de classe comme des lieux à la dynamique fort complexe. Les enseignants doivent en effet faire face à toutes sortes d'incidents à gérer qui se produisent en même temps, situations qui renvoient parfois aux difficultés d'apprentissage, aux rythmes différents des élèves, aux inconduites des uns, aux besoins d'attention des autres. Des événements inattendus, des distractions et des interruptions menacent sans cesse de perturber le cours normal des activités, si bien que les leçons ne se déroulent pas toujours comme prévu. En outre, les enseignants sont pour ainsi dire "sous observation ». La manière dont ils font face aux incidents en classe peut influencer en retour la façon dont les enfants jugent leurs interventions et comment ces derniers y répondront à l'avenir. 
Comme aux échecs, un coup joué par l'un entraîne une réponse de l'autre qui sera suivie d'un autre coup, et ainsi de suite. Doyle (1986) a illustré cette dynamique sophistiquée de la classe par les concepts suivants : la multidimensionnalité, la simultanéité, l'immédiateté, l'imprévisibilité, la visibilité et l'historicité. Plus encore, le travail enseignant implique une composante relationnelle. Pour réussir son mandat d'instruction et d'éducation, l'enseignant doit non seulement entrer en relation avec ses élèves, mais aussi réussir à maintenir cette relation quoi qu'il advienne, et ce, même si les enfants résistent et qu'il doit intervenir pour les ramener à l'ordre.

Le problème de la relation théorie/pratique est ici tragiquement posé. Que faire, quand, d'une part, nous avons besoin d'une théorie complète, d'une sorte d'algorithme comprenant une série finie et non ambiguë d'opérations permettant d'enseigner tout à tous et, d'autre part, nous avons un contexte d'enseignement, la pratique, qui est d'une grande complexité et qui résiste forcément à se laisser quadriller de la sorte? Pour la théorie, nous disposons maintenant de travaux de recherche qui permettent de faciliter l'enseignement des contenus et de gérer la conduite des élèves. L'enseignement explicite est, dans le contexte actuel, l'approche qui semble la mieux fondée pour s'approcher de la théorie fantasmée par Comenius, et encore elle n'en est qu'à des années-lumière. En fait, l'enseignement explicite est l'analogue du plan de match d'une équipe au hockey sur glace. L'entraîneur prévoit des stratégies, mais dans le feu de l'action, dans le contexte d'un chassé-croisé de stratégies échangées avec des adversaires, il doit revoir constamment ce qui a été prévu à la lumière de ce qui se passe.

Que faire donc, quand la théorie est forcément incomplète pour aborder la complexité du réel et que des décisions d'action doivent être prises urgemment ? C'est précisément ici que le concept de tact pédagogique, élaboré pour la première fois par Herbart ${ }^{1}$, prend tout son sens et offre sa plus grande pertinence. Il faut souligner que son œuvre magistrale publiée en allemand n'a cependant pas reçu dans le monde francophone l'accueil qu'elle aurait mérité2. Il convient donc de sortir Herbart, l'espace d'un court texte, d'un injuste oubli.

Comment Herbart définit-il le tact pédagogique? Pour lui, le tact se situe précisément entre la théorie et la pratique. On ne peut, souligne-t-il, s'attendre à déduire de la théorie ce qui doit être fait dans tel cas précis de la pratique, car la théorie «néglige tout le détail, toutes les circonstances individuelles » (2007, p. 24) et, plus encore, « [...] une application aussi parfaite des règles scientifiques exigerait un être surnaturel [...] » (Herbart, 2007, p. 25). Nous n'en sommes pas encore là, n'en déplaise au fantasme coménien! De même, on ne peut se fier davantage à l'expérience, car elle est forcément limitée aux cas rencontrés par un enseignant dans son contexte. Selon Herbart, l'expérience seule engendre forcément la routine au sens où elle est la répétition d'un éventail limité de solutions d'un même individu en réponse aux problèmes rencontrés dans sa situation. En conséquence, « la pratique seule [...] ne donne qu'une expérience extrêmement bornée » (2007, p. 24). Si l'on ne peut chercher du côté de la théorie imparfaite ni du côté trop étroit de l'expérience, il faut donc s'appuyer sur autre chose, sur une sorte de moyen terme entre la théorie et la pratique. Le tact serait cette « faculté rapide de jugement et de décision » (Herbart, 2007, p. 25) et constitue en quelque sorte «le régulateur direct de la pratique » (Herbart, 2007, p. 25). Cette sensibilité à ce qui se passe dans la situation ne donne cependant pas une garantie de la justesse de l'action. Comme le souligne Prairat, en citant Herbart, le tact n'est pas infaillible et on ne saurait en effet « trouver absolument et avec une parfaite justesse [...] ce qu'il y a lieu de faire pour chaque cas individuel (2007, p. 25)» (2017, p. 89). 


\section{CHRONIOLIE}

Le tact en tant que faculté rapide de jugement et de décision ne mobilise pas un processus délibératif qui nécessite d'analyser en profondeur le contexte, de poser un jugement critique, d'inventorier les options possibles, d'en peser le pour et le contre. Même si cela est peut-être parfois nécessaire dans certaines situations comme dans les cas d'inconduites majeurs des élèves, il reste qu'un tel processus de délibération exige trop de temps parce que la situation en temps réel de l'enseignant est parsemée de microdécisions et commande le plus souvent un jugement immédiat et une action prompte. La plupart du temps, il s'agit plutôt de saisir immédiatement, d'un « coup d'œil », ce qui se passe et d'agir. Par exemple, sur le plan de l'apprentissage des contenus, Herbart souligne que : « le tact pédagogique consiste surtout à discerner quand il faut abandonner un élève à son allure lente, et quand il convient, au contraire, d'aller vite» (2007, p. 29). Cette forme de sensibilité immédiate aux choses et aux êtres permet de juger le moment favorable pour intervenir, ce que les Grecs désignaient sous le nom de Kairos.

En outre, cette sensibilité, cette capacité de percevoir sur-le-champ permet de calibrer finement l'agir entre l'excès et le défaut, entre le trop et le trop peu. C'est ce qui ressort à la lecture de l'ouvrage d'Agathon intitulé Les douze vertus d'un bon maître, quand il établit minutieusement la liste des comportements que l'enseignant doit adopter ou éviter pour pratiquer chacune des vertus. On y repère partout un «ni trop ni trop peu » dans ses prescriptions. Le moment favorable, l'intensité nécessaire, le geste approprié, la parole adéquate, l'agir raisonnable, la proximité ou la distance suffisante, la fermeté ou la souplesse adaptée ne renvoient pas à des moyennes statistiques ni à des gestes codés. Ces expressions désignent plutôt la place singulière qu'occupe le tact pédagogique dans le travail de l'enseignant (Bissonnette, Gauthier et Castonguay, 2017).

Comment le tact se développe-t-il? Ici, les propos d'Herbart sont intéressants : le tact se forme d'abord par la pratique au sens où ce que nous apprenons dans l'action exerce une pression sur nos dispositions pour agir. «En d'autres termes : c'est par la méditation, par la réflexion, par la recherche, par la science que l'éducateur doit préparer, non pas tant ses actes futurs dans des cas particuliers [nous ajoutons, ce que la théorie ne peut garantir], que lui-même (nous soulignons) [...].» (2007, p. 26). L'étude et la recherche à la suite de l'action servent donc au maître à former ses propres dispositions à agir, c'est-à-dire son tact. «[...] Dans la pratique même, celui-là seul apprend l'art qui a appris la science auparavant par le travail de la pensée [...] et qui a déterminé à l'avance les impressions futures que l'expérience devait faire sur lui (nous soulignons) »(Herbart, 2007, p. 27).

Agir avec tact en enseignement, c'est donc avoir appris à sentir les nuances d'une situation et être capable d'intervenir avec finesse tout en maintenant la relation avec les élèves afin que leur instruction et leur éducation puissent advenir.

\section{Notes}

1 Herbart (1776-1841), philosophe et pédagogue allemand, successeur de Kant, a publié en 1806 son grand traité de pédagogie, traduit en français en 1894 sous le titre Pédagogie générale déduite du but de l'éducation.

2 Compayré (1903), se référant au Dictionnaire de pédagogie de W. Rein, fait état d'une bibliographie de près de 200 pages et compte pas moins de 2234 livres ou brochures publiés sur lui vers 1900 en Europe! 


\section{Références}

Agathon (1834). Les douze vertus d'un bon maître par M. De La Salle instituteur des Frères des écoles chrétiennes expliquées par le Frère Agathon. Avignon : Séguin Aîné.

Bissonnette, S., Gauthier, C. et Castonguay, M. (2017). L'enseignement explicite des comportements. Pour une gestion efficace des élèves et des comportements en classe et dans l'école. Montréal, QC : Chenelière éducation.

Compayré, G. (1903). Herbart et l'éducation par l'instruction. Paris : Paul Delaplane.

Herbart, J. F. (2007). Tact, autorité, expérience et sympathie en pédagogie. Paris : Économica.

\section{Pour citer cet article}

Gauthier, C. (2019). Le tact pédagogique. Formation et profession. 27(3), 121-124.

http://dx.doi.org/10.18162/fp.2019.a187 